PROCEEDINGS OF THE

AMERICAN MATHEMATICAL SOCIETY

Volume 125, Number 3, March 1997, Pages 795-799

S 0002-9939(97)03618-6

\title{
CONTINUOUS FELL BUNDLES ASSOCIATED TO MEASURABLE TWISTED ACTIONS
}

\author{
RUY EXEL AND MARCELO LACA
}

(Communicated by Palle E. T. Jorgensen)

\begin{abstract}
Given a measurable twisted action of a second-countable, locally compact group $G$ on a separable $C^{*}$-algebra $A$, we prove the existence of a topology on $A \times G$ making it a continuous Fell bundle, whose cross sectional $C^{*}$ algebra is isomorphic to the Busby-Smith-Packer-Raeburn crossed product.
\end{abstract}

\section{INTRODUCTION}

Let $A$ be a $C^{*}$-algebra and $G$ be a locally compact group. According to [2], a twisted action of $G$ on $A$ is a pair $(\theta, w)$ of maps $\theta: G \rightarrow A u t(A)$, and $w: G \times G \rightarrow$ $\mathcal{U M}(A)$, where $\operatorname{Aut}(A)$ denotes the automorphism group of $A$ and $\mathcal{U M}(A)$ is the set of unitary elements in the multiplier algebra $\mathcal{M}(A)$, satisfying

(i) $\theta_{e}$ is the identity automorphism of $A$,

(ii) $\theta_{r}\left(\theta_{s}(a)\right)=w(r, s) \theta_{r s}(a) w(r, s)^{*}$,

(iii) $w(e, t)=w(t, e)=1$,

(iv) $\theta_{r}(w(s, t)) w(r, s t)=w(r, s) w(r s, t)$,

for $r, s, t$ in $G$ and $a$ in $A$.

Also following [2], we will say that $(\theta, w)$ is measurable if $\theta$ is strongly measurable in the sense that for each $a$ in $A$, the map

$$
t \in G \mapsto \theta_{t}(a) \in A
$$

is Borel measurable, and if $w$ is strictly measurable in the sense that, for each $a$ in $A$, the maps

$$
\begin{gathered}
(r, s) \in G \times G \mapsto a w(r, s) \in A, \\
(r, s) \in G \times G \mapsto w(r, s) a \in A
\end{gathered}
$$

are Borel measurable.

For convenience, we will restrict ourselves to the treatment of separable $C^{*}$ algebras and second countable groups, as in [2], so that, in particular, the various notions of measurability for $A$ valued maps coincide.

Finally, $(\theta, w)$ will be termed continuous if the maps (1)-(3) above are continuous. In this case, it is easy to see that the conditions of [3, Definition 3.8] are

Received by the editors August 22, 1995.

1991 Mathematics Subject Classification. Primary 46L05.

The first author was partially supported by CNPq, Brazil

The second author was supported by the Australian Research Council.

(C)1997 American Mathematical Society 
satisfied, and hence that we can construct the associated semi-direct product bundle of $A$ by $G$ [3, Theorem 3.10].

Fell bundles, also frequently referred to as $C^{*}$-algebraic bundles, were introduced by J. M. G. Fell [4] (see also [5]) in the 60's. Among the many interesting features surrounding this concept, we would like to point out its relevance for the study of crossed product $C^{*}$-algebras. In fact Fell bundles can (and should) be viewed as intermediate steps in the construction of crossed products; the procedure being to start by constructing the associated semi-direct product bundle [5, VIII.4], [3] and then to consider its cross sectional algebra [5, VIII.17.2].

The available theory of Fell bundles, of which [5] is one of the most authoritative accounts, does not include, as far as we know, a systematic study of measurable (as opposed to continuous) bundles. However, crossed products by measurable twisted actions have been profitably studied by Packer and Raeburn [8], where they play an important role in the theory of group actions on $C^{*}$-algebras. Therefore, it seems plausible that this latter crossed product construction could be obtained in a similar two step procedure, involving, as the intermediate step, the construction of a "measurable" Fell bundle.

Our main point, however, is that, given a measurable twisted action of a secondcountable group on a separable algebra, the associated $L^{1}$ algebra studied by Busby-Smith [2], as well as the crossed product of Packer-Raeburn [8], can both be obtained from a continuous Fell bundle. This result bears a certain degree of similarity with the result of S. Banach, according to which a measurable homomorphism between complete metric groups is necessarily continuous $[1$, Theorem I.4].

The stabilisation trick of Packer and Raeburn can be used to obtain information on the representation theory of twisted crossed products. Indeed, Kaliszewski $[6,7]$, has developed a theory of induced representations and Mackey normal subgroup analysis suitable for measurable twisted systems, as had been suggested in the introduction to [8]. Since the Mackey machine has, to a large extent, been generalized to Fell bundles, (e.g. [5, chapters XI and XII]), our result gives a new way of making it available to the study of twisted crossed products by establishing that they are isomorphic to cross sectional algebras of Fell bundles.

The present work was largely developed during the first author's visit to the Mathematics Department at the University of Newcastle, whose members he would like to thank for their warm hospitality.

\section{The MAIN RESUlts}

Let us fix, throughout, a measurable twisted action $(\theta, w)$ of a locally compact, second-countable group $G$ on a separable $C^{*}$-algebra $A$. If we let $G^{d}$ denote the discrete group obtained by replacing the given topology of $G$ by the discrete topology, then, following [3, Theorem 2.7], we get a Fell bundle structure on $A \times G^{d}$, by means of the following operations, where we use the notation $a \delta_{t}$ for $(a, t)$ in $A \times G^{d}$ :

$$
\left(a \delta_{r}\right)\left(b \delta_{s}\right)=a \theta_{r}(b) w(r, s) \delta_{r s}, \quad a, b \in A, \quad r, s \in G,
$$

and

$$
\left(a \delta_{t}\right)^{*}=\theta_{t}^{-1}\left(a^{*}\right) w\left(t^{-1}, t\right)^{*} \delta_{t^{-1}}, \quad a \in A, \quad t \in G^{d}
$$


We will refer to this bundle as $\mathfrak{B}\left(A, G^{d}, \theta, w\right)$. Of course it bears no relationship, whatsoever, with the topological nature of $G$. Our main result is precisely intended to fill this gap. We will therefore show that there exists a topology on $A \times G$ for which $\mathfrak{B}\left(A, G^{d}, \theta, w\right)$ is a continuous Fell bundle over $G$. (Whenever $G$ occurs without the superscript ' $d$ ', it is to be thought of as carrying its originally given topology.) In addition, we will show that the Banach algebra of $L^{1}$ sections of $\mathfrak{B}\left(A, G^{d}, \theta, w\right)$ is isomorphic to $L^{1}(G, A, \theta, w)$ (see [2]) and its cross sectional $C^{*}$-algebra is isomorphic to the Packer-Raeburn crossed product $A \rtimes_{\theta, w} G$, both isomorphisms being canonical.

One of the main ingredients in the proof of this result is the "Packer-Raeburn stabilization trick" [8, Theorem 3.4], which we briefly describe below, mainly to fix our notation.

2.1. Definition ([8], Definition 3.1). The measurable twisted actions $(\alpha, u)$ and $(\beta, w)$ of $G$ on $A$ are exterior equivalent if there exists a strictly measurable map $v: G \rightarrow \mathcal{U M}(A)$ such that

(i) $\beta_{t}(a)=v_{t} \alpha_{t}(a) v_{t}^{*}, \quad a \in A, \quad t \in G$.

(ii) $w(r, s)=v_{r} \alpha_{r}\left(v_{s}\right) u(r, s) v_{r s}^{*}, \quad r, s \in G$.

2.2. Proposition. If $(\alpha, u)$ and $(\beta, w)$ are exterior equivalent then the associated bundles $\mathfrak{B}\left(A, G^{d}, \alpha, u\right)$ and $\mathfrak{B}\left(A, G^{d}, \beta, w\right)$ are isomorphic.

Proof. One checks that the map $\phi: A \times G \rightarrow A \times G$ defined by $\phi(a, t)=\left(a v_{t}^{*}, t\right)$ is an isomorphism for the respective bundle structures. In proving this, the identity $\beta_{t}^{-1}\left(v_{t}\right)=\alpha_{t}^{-1}\left(v_{t}\right)$, which follows from 2.1(i) with $a=\alpha_{t}^{-1}\left(v_{t}\right)$, comes in handy.

The Packer-Raeburn stabilization trick asserts that, if $(\theta, w)$ is a measurable twisted action of the second-countable group $G$ on the separable $C^{*}$-algebra $A$, then there exists a strongly continuous (untwisted) action $\beta$ of $G$ on $A \otimes \mathcal{K}$ (where $\mathcal{K}$ denotes the algebra of compact operators on a separable Hilbert space), such that $(\theta \otimes 1, w \otimes 1)$ is exterior equivalent to $(\beta, 1)$. Therefore, by $(2.2)$, the bundles

$$
\mathfrak{B}_{0}:=\mathfrak{B}\left(A \otimes \mathcal{K}, G^{d}, \theta \otimes 1, w \otimes 1\right)
$$

and

$$
\mathfrak{B}_{1}:=\mathfrak{B}\left(A \otimes \mathcal{K}, G^{d}, \beta, 1\right)
$$

are isomorphic. Now, since $\beta$ is strongly continuous, the product topology on $(A \otimes \mathcal{K}) \times G$ makes $\mathfrak{B}_{1}$ a continuous Fell bundle over $G$ [3, Theorem 3.10]. Thus we can make $\mathfrak{B}_{0}$ into a continuous Fell bundle over $G$ by transferring the topology from $\mathfrak{B}_{1}$ to $\mathfrak{B}_{0}$ via the isomorphism given by $(2.2)$.

On the other hand, if $p$ denotes a minimal projection in $\mathcal{K}$, then $\mathfrak{B}\left(A, G^{d}, \theta, w\right)$ sits naturally in $\mathfrak{B}_{0}$ as the sub-bundle $(A \otimes p) \times G^{d}$.

The crucial point in our argument is to show that this sub-bundle, with the inherited topology, is a continuous Fell bundle.

In order to verify this claim, let us fix some notation. First of all, given the two distinct bundle structures on $(A \otimes \mathcal{K}) \times G^{d}$, let us agree to denote the element $(x, t)$ in $(A \otimes \mathcal{K}) \times G^{d}$ by $x \partial_{t}$ when it is viewed as an element of $\mathfrak{B}_{1}$ while retaining the notation $x \delta_{t}$ when $\mathfrak{B}_{0}$ is concerned. Secondly, let us denote by $v$ a given Borel map $v: G \rightarrow \mathcal{U M}(A \otimes \mathcal{K})$ implementing the equivalence (2.1) between $(\theta \otimes 1, w \otimes 1)$ and $(\beta, 1)$, which exists by the stabilization trick. 
By Proposition (2.2), the map

$$
\phi\left(x \delta_{t}\right)=x v_{t}^{*} \partial_{t}, \quad x \in A \otimes \mathcal{K}, \quad t \in G,
$$

is an isomorphism from $\mathfrak{B}_{0}$ to $\mathfrak{B}_{1}$.

Recall that any multiplier of the unit fiber algebra of a Fell bundle extends to a multiplier of order $e$ (where $e$ denotes the group unit) of the bundle concerned [5, VIII.3.8]. In particular, $1 \otimes p$, viewed as a multiplier of $A \otimes \mathcal{K}$, extends to a multiplier of $\mathfrak{B}_{1}$, which we will denote by $\pi$.

2.3. Lemma. For each $t$ in $G$ one has

$$
\phi\left((A \otimes p) \delta_{t}\right)=\pi\left((A \otimes \mathcal{K}) \partial_{t}\right) \pi .
$$

Proof. We have

$$
\begin{aligned}
\pi\left((A \otimes \mathcal{K}) \partial_{t}\right) \pi & =(1 \otimes p) \partial_{e}(A \otimes \mathcal{K}) \partial_{t}(1 \otimes p) \partial_{e} \\
& =(1 \otimes p)(A \otimes \mathcal{K}) \beta_{t}(1 \otimes p) \partial_{t}=(1 \otimes p)(A \otimes \mathcal{K}) v_{t}\left(\theta_{t} \otimes 1\right)(1 \otimes p) v_{t}^{*} \partial_{t} \\
& =(1 \otimes p)(A \otimes \mathcal{K}) v_{t}(1 \otimes p) v_{t}^{*} \partial_{t}=(A \otimes p) v_{t}^{*} \partial_{t}=\phi\left((A \otimes p) \delta_{t}\right) .
\end{aligned}
$$

This brings us to our first main result.

2.4. Theorem. Let $(\theta, w)$ be a measurable twisted action of the locally compact, second-countable group $G$ on the separable $C^{*}$-algebra $A$. Also let $\mathfrak{B}_{1}$ be as above. Then $\mathfrak{B}\left(A, G^{d}, \theta, w\right)$, equipped with the topology induced by the embedding

$$
\mathfrak{B}\left(A, G^{d}, \theta, w\right) \rightarrow \mathfrak{B}_{1}
$$

given by

$$
a \delta_{t} \mapsto(a \otimes p) v_{t}^{*} \partial_{t},
$$

is a continuous Fell bundle over $G$.

Proof. The proof consists in showing that the collection of subspaces

$$
(A \otimes p) v_{t}^{*} \partial_{t} \subseteq(A \otimes \mathcal{K}) \partial_{t}, \quad t \in G,
$$

forms a continuous bundle of Banach spaces over $G$ [5, II.13.1], in the sense that one can find a continuous section $\gamma$ passing through any preassigned element $(a \otimes p) v_{t_{0}}^{*} \partial_{t_{0}}$, and such that $\gamma(t) \in(A \otimes p) v_{t}^{*} \partial_{t}$ for all $t$ in $G$ [5, II.13.18], [3, Proposition 3.3]. For this, it suffices to take a section $\sigma$ of $\mathfrak{B}_{1}$ such that $\sigma\left(t_{0}\right)=(a \otimes p) v_{t_{0}}^{*} \partial_{t_{0}}$. Since the left and right actions of multipliers are continuous maps on the bundle [5, VIII.2.14]), $\gamma(t)=\pi \sigma(t) \pi$ gives the desired section.

Let us denote by $\mathfrak{B}(A, G, \theta, w)$ (omitting the superscript in $G^{d}$ ), the continuous bundle over $G$, arising from $(2.4)$

2.5. Theorem. Let $A, G, \theta$ and $w$ be as in (2.4). Then the formula $\psi(f)(t)=$ $f(t) \delta_{t}$, for $f$ in $L^{1}(G, A)$ and $t$ in $G$, gives a Banach ${ }^{*}$-algebra isomorphism

$$
\psi: L^{1}(G, A, \theta, w) \rightarrow L^{1}(\mathfrak{B}(A, G, \theta, w)),
$$

which, in turn, induces an isomorphism between the crossed product $A \rtimes_{\theta, w} G$ and the cross sectional $C^{*}$-algebra of $\mathfrak{B}(A, G, \theta, w)$. 
Proof. Under the identification provided by Theorem (2.4) we may write $\psi(f)(t)=$ $(f(t) \otimes p) v_{t}^{*} \partial_{t}$. Observe that, if $f$ is in $L^{1}(G, A, \theta, w)$ then $\psi(f)$ is an integrable section of $\mathfrak{B}(A, G, \theta, w)$ because $v$ is strictly Borel measurable. The same reasoning applies to prove the converse and hence $\psi$ gives an isometric linear isomorphism

$$
\psi: L^{1}(G, A, \theta, w) \rightarrow L^{1}(\mathfrak{B}(A, G, \theta, w)) .
$$

It is now easy to show that $\psi$ is also a Banach *-algebra isomorphism.

The description of $A \rtimes_{\theta, w} G$ which better suits our purposes is that given in [8, Remark 2.6], where $A \rtimes_{\theta, w} G$ is described as the enveloping $C^{*}$-algebra of $L^{1}(G, A, \theta, w)$. Since, on the other hand, the cross sectional $C^{*}$-algebra of $\mathfrak{B}(A, G, \theta, w)$ is the enveloping $C^{*}$-algebra of the algebra of integrable sections of this bundle, we see that the last part of the statement follows by taking the enveloping $C^{*}$-algebras of the corresponding Banach algebras.

\section{REFERENCES}

1. S. Banach, Théorie des Opérations Linéaires, Hafner Publishing Co., New York, 1932. MR 17:175h

2. R. C. Busby and H. A. Smith, Representations of Twisted Group Algebras, Trans. Amer. Math. Soc. 149, (1970), 503-537. MR 41:9013

3. R. Exel, Twisted Partial Actions, A Classification of Stable $C^{*}$-Algebraic Bundles, Universidade de São Paulo, 1994, preprint; Proc. London Math. Soc. (to appear).

4. J. M. Fell, An extension of Mackey's method to Banach *-Algebraic Bundles, Memoirs Amer. Math. Soc., vol. 90, 1969. MR 41:4255

5. J. M. G. Fell and R. S. Doran, Representations of *-algebras, locally compact groups, and Banach *-algebraic bundles, Pure and Applied Mathematics series, vol. 125 and 126, Academic Press, 1988. MR 90c:46001; MR 90c: 46002

6. S. Kaliszewski, A note on Morita equivalence of twisted $C^{*}$-dynamical systems, Proc. Amer. Math. Soc. 123, (1995), 1737-1740.

7. S. Kaliszewski, Induced representations of twisted $C^{*}$-dynamical systems, University of Newcastle, 1995, preprint.

8. J. A. Packer and I. Raeburn, Twisted crossed products of $C^{*}$-algebras, Math. Proc. Cambridge Philos. Soc. 106 (1989), 293-311. MR 90g:46097

Departamento de Matemática, Universidade de São Paulo, Rua do Matão, 1010, 05508-900 São Paulo, Brazil

E-mail address: exel@ime.usp.br

Mathematics Department, University of Newcastle, Newcastle, New South Wales 2308, Australia

E-mail address: marcelo@math.newcastle.edu.au 